\title{
Simulação hidrológica em uma bacia hidrográfica representativa dos Latossolos na região Alto Rio Grande, MG
}

\author{
Márcio A. Andrade', Carlos R. de Mello² \& Samuel Beskow ${ }^{3}$
}

\begin{abstract}
RESUMO
A simulação hidrológica consiste de uma importante ferramenta para subsidiar a gestão dos recursos hídricos em bacias hidrográficas. A bacia hidrográfica em estudo está localizada na região Alto Rio Grande, sul do estado de Minas Gerais, drenando uma área de $32 \mathrm{~km}^{2}$ diretamente para o reservatório da Usina Hidrelétrica de Camargos (UHE - Camargos/Cemig) conhecida como Bacia Hidrográfica do Ribeirão Jaguara (BHRJ). Neste trabalho objetivou-se calibrar e validar o modelo SWAT (Soil and Water Assessment Tool) para a simulação do escoamento superficial na BHRJ. Para isto, o modelo requer mapas georreferenciados de uso atual do solo, unidades pedológicas e modelo digital de elevação, além de dados meteorológicos e hidrológicos. Para este estudo foi aplicada uma série histórica de vazões e dados climáticos diários de 01/01/2006 a 31/08/2009. A acurácia do modelo foi medida com base no coeficiente de Nash-Sutcliffe $\left(C_{N S}\right)$ tendo-se obtido valores de 0,66 e 0,87 para as fases de calibração e validação, respectivamente. De acordo com a classificação proposta para o modelo SWAT e com base nos valores de $\mathrm{C}_{\mathrm{NS}}$ como referência, o modelo pode ser considerado adequado para simulação do comportamento hidrológico da BHRJ, a qual é representativa dos latossolos na região Alto Rio Grande, sul de Minas Gerais.
\end{abstract}

Palavras-chave: modelo hidrológico, SWAT, escoamento superficial, balanço hídrico

\section{Hydrological simulation in a watershed with predominance of Oxisol in the Upper Grande river region, MG - Brazil}

\begin{abstract}
Hydrological simulation has been considered an important tool applied to support water resources management in watersheds. The studied watershed is located in the Upper Grande river region in southern Minas Gerais State, draining an area of $32 \mathrm{~km}^{2}$ towards the Camargos Hydroelectric Power Plant Reservoir (UHE - Camargos/CEMIG). In this context, this study aimed to calibrate and validate the SWAT model (Soil and Water Assessment Tool) for assessing quantitatively the stream flow in the Jaguara Creek Watershed (JCW). For that, SWAT model requires land-use and soil maps and digital elevation model, besides historical series related to meteorological and hydrological data sets. January 1, 2006 to August 31, 2009 was the period used for the above-mentioned historical series. The performance of SWAT was measured through NashSutcliffe coefficient $\left(\mathrm{C}_{\mathrm{NS}}\right)$ which was 0.66 and 0.87 for calibration and validation, respectively. Comparing $\mathrm{C}_{\mathrm{NS}}$ results found in this study to the ones established in a SWAT model performance classification, it was found that such model can be used to evaluate the hydrological behavior in the JCW, which is a watershed representative of the Oxisol in the Upper Grande river region in southern Minas Gerais State.
\end{abstract}

Key words: hydrological model, SWAT, streamflow, water balance 


\section{INTRODUÇÃO}

As ações antrópicas são responsáveis por modificações consideráveis no ambiente de bacias hidrográficas, tendo em vista sua influência quantitativa e qualitativa direta sobre os processos hidrológicos. De forma geral, o resultado final dessas modificações é percebido apenas quando da ocorrência de eventos hidrológicos extremos e de suas consequências, tais como, estiagens severas, inundações e destruições, produção e transporte de sedimentos em bacias hidrográficas, prejudicando drasticamente a agricultura além da possibilidade de aumento no aporte de nutrientes em mananciais com influência direta na qualidade da água.

A realização de estudos hidrológicos em bacias hidrográficas surge da necessidade de se compreender o funcionamento do balanço hídrico, dos processos que controlam o movimento da água e seus prováveis impactos sobre a quantidade e a qualidade da água. $\mathrm{O}$ modelo hidrológico é uma ferramenta desenvolvida para avaliação dos processos hidrológicos em diferentes escalas espaciais e temporais (Spruill et al., 2000). Wagener et al. (2004) relatam que este tipo de modelo pode ser aplicado para avaliar estratégias de gerenciamento de recursos hídricos, a reposta de bacias hidrográficas a variações climáticas periódicas, cheias de projeto, cheias em tempo real e condições de contorno para modelos de circulação atmosférica. Diversos modelos hidrológicos têm sido desenvolvidos e utilizados em diferentes partes do mundo e com variadas finalidades práticas como, por exemplo: SMAP (Saraiva et al., 2011), LASH (Beskow et al., 2011b), annAGNPS (Yuan et al., 2011) e MGB/ IPH (Collischonn et al., 2007) dentre outros.

Com o advento dos Sistemas de Informações Geográficas (SIG) os modelos hidrológicos têm sido desenvolvidos e aplicados com maior embasamento físico uma vez que o uso de um SIG possibilita a representação dos parâmetros dos modelos, de forma distribuída (Veith et al., 2010). Além disso, Vieux (2004) relata que a integração da hidrologia ao SIG é absolutamente natural, haja vista que os SIGs são capazes de capturar, armazenar, manipular, analisar e visualizar variados conjuntos de dados georreferenciados e a hidrologia é uma ciência que apresenta um caráter espacial, desta forma, os modelos hidrológicos distribuídos demandam, em geral, grandes quantidades de dados; no entanto, os modelos são representações inexatas do movimento da água em um sistema natural, razão pela qual devem ser calibrados com os dados observados. Enquanto a calibração minimiza os erros entre a saída do modelo e os dados observados, este processo se torna complicado em virtude do grande número de parâmetros não mensuráveis que precisam ser estimados (Veith et al., 2010).

O modelo hidrológico SWAT (Soil and Water Assessment Tool) foi desenvolvido na década de 90, nos Estados Unidos, pelo Agricultural Research Service e pela Texas A \& M University. O SWAT é um modelo matemático que permite que diferentes processos físicos sejam simulados em uma bacia hidrográfica (Arnold \& Allen, 1996). O SWAT tem sido adaptado, desde o seu surgimento, para algumas áreas específicas e foi integrado ao SIG possibilitando a entrada do banco de dados, a elaboração e edição de cenários ambientais e sua representação em forma de gráficos e mapas, controle e simulações, além de extrair e organizar as saídas do modelo (Marchioro, 2008). Por meio de uma análise na literatura existente foi possível constatar algumas aplicações do SWAT em bacias hidrográficas brasileiras, como os de Pinto (2011), Silva et al. (2011), Durães et al. (2011) e Baltokoski et al. (2010) dentre outros.

No contexto acima objetivou-se, com este trabalho, aplicar e testar o modelo hidrológico SWAT, seguindo uma sequência de procedimentos recomendada (análise de sensibilidade, calibração e validação), na bacia hidrográfica do ribeirão Jaguara, a qual é representativa dos Latossolos na região do Alto Rio Grande, sul de Minas Gerais, visando obter resultados associados à viabilidade do uso deste modelo, na referida região.

\section{Material E MÉTODOS}

A Bacia Hidrográfica do Ribeirão Jaguara (BHRJ) possui uma área de drenagem de $32 \mathrm{~km}^{2}$, a qual deságua diretamente no reservatório da usina hidrelétrica (UHE) de Camargos/ CEMIG. A BHRJ tem uma ocupação predominantemente rural e apresenta características pedológicas, hidrológicas e de ocupação dos solos, típicas da região do Alto Rio Grande. Estudos que visam avaliar o comportamento desta bacia são relevantes na compreensão da dinâmica do ciclo hidrológico e no auxílio à tomada de decisões, no que tange ao uso, conservação e preservação da qualidade dos recursos naturais nesta região. Neste sentido, alguns trabalhos foram desenvolvidos na referida bacia, como os de Beskow et al. (2011a, b) e Gomes et al. (2007).

O clima, de acordo com classificação de Köppen, é Cwa, caracterizado por verões amenos e úmidos e invernos frios e secos, com temperatura média anual de $19{ }^{\circ} \mathrm{C}$ e precipitação média anual de $1500 \mathrm{~mm}$ (Mello et al., 2012).

Para o presente estudo as variáveis meteorológicas de interesse foram monitoradas a cada $30 \mathrm{~min}$ por meio de uma estação meteorológica automática, com destaque para precipitação, temperatura e umidade relativa do ar, velocidade e direção do vento e radiação solar. Obteve-se a série de dados de vazão a partir de monitoramento fluviométrico conduzido por meio de um linígrafo automático e da utilização de uma curva-chave estabelecida através de campanhas hidrológicas, realizadas durante as estações secas e úmidas, entre os anos de 2007 e 2009, destinadas a medições de descarga líquida (vazão) e o nível d'água correspondente. Os dados coletados foram referentes ao período de 01 de janeiro de 2006 a 31 de agosto de 2009, constituindo a base de dados temporal para os devidos testes com o modelo SWAT nesta bacia.

A fim de caracterizar o uso e a ocupação do solo na bacia (Figura 1A) foi utilizada uma imagem do satélite ALOS, de maio de 2008, que fornece imagens multiespectrais com resolução de $10 \mathrm{~m}$. Após a interpretação desta imagem de satélite utilizando-se ferramentas de processamento digital de imagens (PDI) em conjunto com verdades de campo, as quais foram coletadas por meio de dispositivos GPS, foi possível constatar, na BHRJ, a presença de eucalipto $(7,68 \%)$, café $(4,11 \%)$, solo exposto $(9,63 \%)$, milho $(22,85 \%)$, vegetação nativa $(13,13 \%)$ e pastagem $(42,60 \%)$. Obteve-se o modelo digital de elevação (DEM) (Figura 1B) por meio de interpolação 
A.

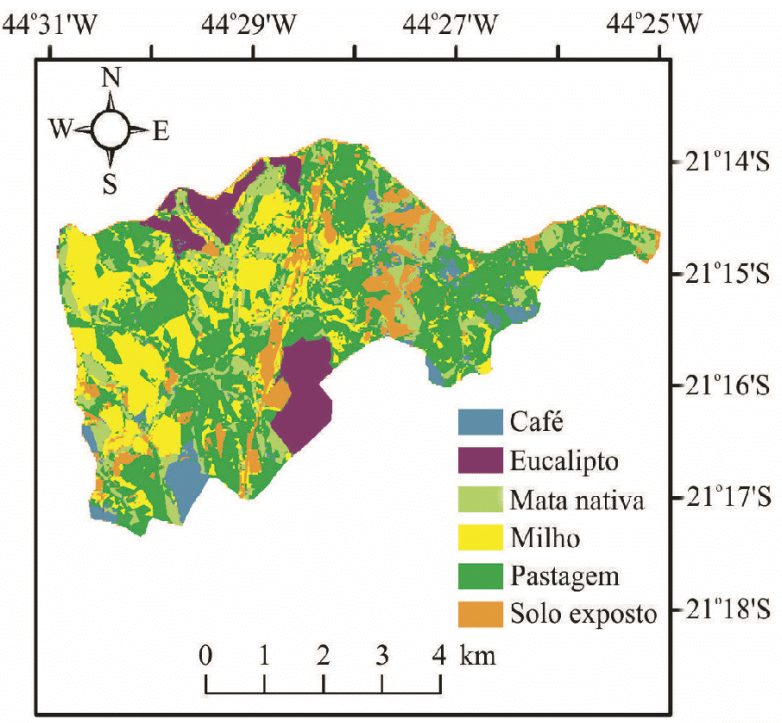

B. $44^{\circ} 31^{\prime} \mathrm{W}$

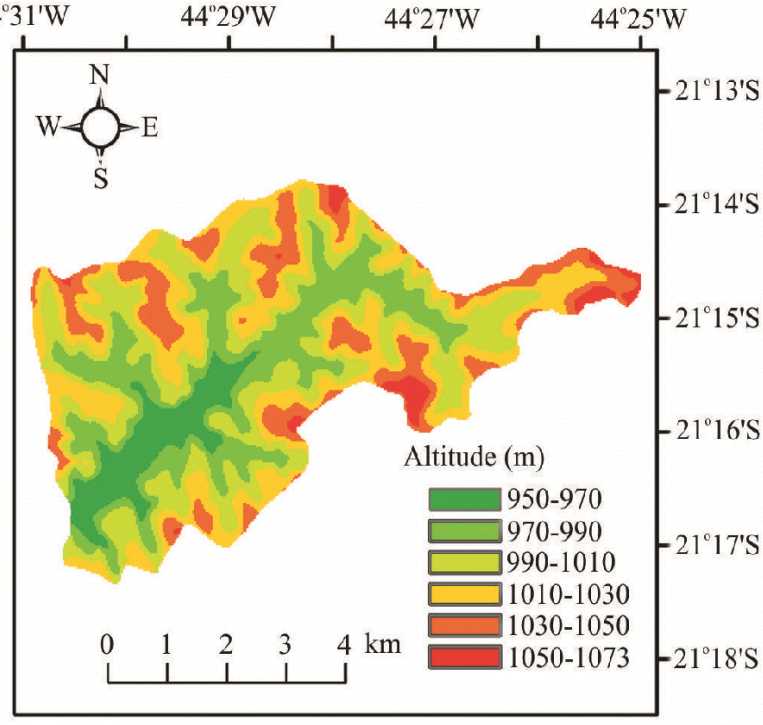

C. $44^{\circ} 31^{\prime} \mathrm{W}, \quad 44^{\circ} 29^{\prime} \mathrm{W}, \quad 44^{\circ} 27^{\prime} \mathrm{W}, \quad 44^{\prime} 25^{\prime} \mathrm{W}$

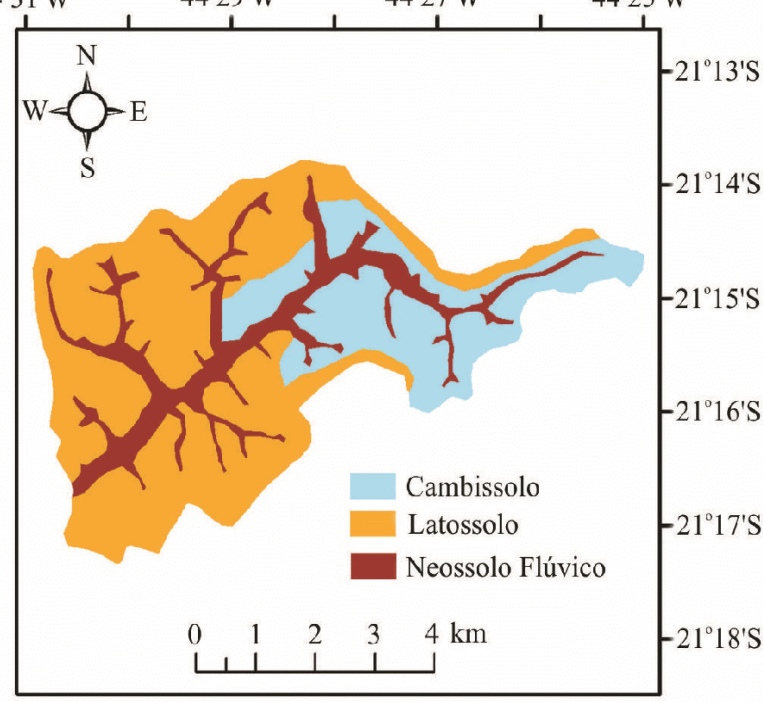

Figura 1. Mapas de uso e ocupação do solo (A), Modelo digital de elevação (B) e Solos (C) da BHRJ para a aplicação no SWAT de um mapa obtido junto ao Instituto Brasileiro de Geografia e Estatística (IBGE) que disponibiliza informações sobre curvas de nível, pontos cotados e hidrografia. Para obtenção do DEM, esses procedimentos de interpolação foram realizados a partir de ferramentas disponíveis em Sistemas de Informações Geográficas (SIG), mais especificamente o ArcGIS. Os valores de altitude variam de 956 a 1073 m e as declividades são consideravelmente íngremes em alguns trechos da bacia (até 46,9\%); seu valor médio é de $11,6 \%$. O mapa de solos (Figura 1C) possui a seguinte distribuição percentual (Araújo, 2006): a) Latossolos em 59,8\% da área; b) Cambissolos em 23,4\% da área; c) Neossolos Flúvicos em 16,8\% da área, encontrados ao longo da rede de drenagem cuja paisagem tem topografia quase plana.

O SWAT é um modelo baseado em características físicas das bacias hidrográficas, computacionalmente eficiente para operar tanto em pequenas quanto em grandes bacias e é contínuo no tempo, sendo capaz de simular longos períodos (Santhi et al., 2001). O modelo requer, necessariamente, três diferentes arquivos na forma de mapas: modelo digital de elevação, mapas de solos e de uso do solo (Figura 1). Além desses mapas o SWAT também necessita de dados tabulares referentes às condições climáticas: dados diários de precipitação, temperatura máxima e mínima do ar, radiação solar, velocidade do vento e umidade relativa (Gassman et al., 2007).O método nele selecionado para simulação da componente evapotranspiração foi o de Penman-Monteith. Salienta-se que o modelo SWAT também disponibiliza outras opções para o módulo evapotranspiração, como a inserção dos próprios valores de evapotranspiração potencial e a utilização dos métodos de Hargreaves e Priestley-Taylor (Neitsch et al., 2005).

O modelo considera quatro volumes de controle para basear sua estrutura no balanço hídrico, ou seja,os reservatórios superficial, subsuperficial, subterrâneo raso ou livre e o subterrâneo profundo. O balanço hídrico fica assim representado (Eq. 1):

$$
\mathrm{SW}_{\mathrm{t}}=\mathrm{SW}_{0}+\sum\left(\mathrm{R}_{\mathrm{i}}-\mathrm{Q}_{\text {sup }_{\mathrm{i}}}-\mathrm{ET}_{\mathrm{i}}-\mathrm{P}_{\mathrm{i}}-\mathrm{QL}_{\mathrm{i}}\right)
$$

em que:

$\mathrm{SW}_{\mathrm{t}}$ - conteúdo final de água no solo, $\mathrm{mm}$

$\mathrm{SW}_{0}$ - conteúdo de água no solo disponível, $\mathrm{mm}$

$\mathrm{t} \quad$ - tempo, dias

$\mathrm{R}_{\mathrm{i}} \quad$ - precipitação, mm

$\mathrm{Q}_{\text {supi }}$ - escoamento superficial, $\mathrm{mm}$

$\mathrm{ET}_{\mathrm{i}}$ - evapotranspiração real, mm

$\mathrm{P}_{\mathrm{i}} \quad$ - percolação da água na camada simulada para a camada inferior, $\mathrm{mm}$

$\mathrm{QL}_{\mathrm{i}}$ - escoamento lateral, $\mathrm{mm}$

O SWAT permite a divisão da bacia em sub-bacias, as quais podem ser, ainda, parametrizadas, usando-se o conceito de unidades de resposta hidrológica (Hydrologic Response Units - HRU's), permitindo representar as diferenças entre os tipos de solo, cobertura vegetal e topografia. Subdividir a bacia em áreas contendo combinações únicas possibilita ao modelo 
capturar diferenças na evapotranspiração e em outras condições hidrológicas para diferentes culturas e solos (Gassman et al., 2007).

Neste trabalho foi adotada, para a geração das HRU's, uma divisão que contemple a combinação entre as classes de solos presentes na bacia em conjunto com suas respectivas declividades. Com isto, foi fez-se uma divisão para que a classe de declividade de $0-18 \%$ represente os Latossolos e os Neossolos enquanto a classe de declividade de 18-35\% inclua os Cambissolos. A última classe e de menor representatividade foi aquela com declividades maiores que 35\%. Quanto ao uso do solo foi definido que apenas as classes que ocupassem uma área superior a $10 \%$ da área total da bacia fossem consideradas para as definições das HRU's; o que se deve ao fato de que esses usos são os mais influentes no balanço hídrico da bacia, tanto no escoamento quanto na evapotranspiração. Com o exposto acima foram obtidas 27 HRU's.

A análise de sensibilidade é um instrumento para previsão dos parâmetros do modelo sobre os resultados de sua saída, o que possibilita a racionalização da etapa de calibração, tal como, também, a fixação dos parâmetros para os quais o modelo é pouco sensível (Durães et al., 2011). Em referência à análise de sensibilidade, o modelo SWAT utiliza uma combinação do "Latin Hypercube"com o "One-Factor-At-a-Time" (Griensven, 2005). O SWAT possui 21 parâmetros que exercem influência na simulação da vazão (Tabela 1); desta forma, um número tão grande de parâmetros se torna inviável, necessitando da sua redução para tentar seguir o princípio da parcimônia (Muleta \& Nicklow, 2005).

Tabela 1. Parâmetros calibráveis pelo modelo SWAT na simulação da variável hidrológica vazão

\begin{tabular}{|c|c|}
\hline Parâmetro & Descrição \\
\hline SOL_AWC & Armazenamento de água no solo ( $\left.\mathrm{mm} \mathrm{mm}^{-1}\right)$ \\
\hline SOL_K & Condutividade hidráulica do solo saturado $\left(\mathrm{mm} \mathrm{h}^{-1}\right)$ \\
\hline ALPH̄A_BF & Constante de recessão do escoamento debase (dias) \\
\hline GWQMN & $\begin{array}{l}\text { Nível limite da água no aquífero raso para a ocorrência do } \\
\text { fluxo de base }(\mathrm{mm})\end{array}$ \\
\hline GW_REVAP & $\begin{array}{l}\text { Coeficiente de ascensão da água à zona de saturação } \\
\text { (adimensional) }\end{array}$ \\
\hline REVAPMN & $\begin{array}{l}\text { Profundidade limite da água no solo para a ocorrência da } \\
\text { ascensão da água à zona não saturada (mm) }\end{array}$ \\
\hline GW_DELAY & Intervalo de tempo para a recarga do aquífero (dias) \\
\hline EPCO & $\begin{array}{l}\text { Coeficiente de compensação de absorção de água pelas } \\
\text { plantas (adimensional) }\end{array}$ \\
\hline RCHRG_DP & $\begin{array}{l}\text { Coeficiente de percolação da água para o aquífero } \\
\text { profundo }(\mathrm{mm})\end{array}$ \\
\hline SOL_Z & Profundidade da camada do solo (mm) \\
\hline CN2 & $\begin{array}{l}\text { Curva Número inicial para a condição de umidade AMC II } \\
\text { (adimensional) }\end{array}$ \\
\hline SURLAG & $\begin{array}{l}\text { Coeficiente de retardamento do escoamento superficial } \\
\text { direto }\end{array}$ \\
\hline CH_N2 & $\begin{array}{l}\text { Coeficiente de Manning para o canal pri ncipal } \\
\text { (adimensional) }\end{array}$ \\
\hline SLOPE & Declividade média da sub-bacia $\left(\mathrm{m} \mathrm{m}^{-1}\right)$ \\
\hline $\begin{array}{l}\text { SLSUBBSN } \\
\text { BLAI }\end{array}$ & $\begin{array}{l}\text { Comprimento médio da encosta da sub -bacia }(\mathrm{m}) \\
\text { Índice de área foliar máximo }\left(\mathrm{m}^{2} \mathrm{~m}^{-2}\right)\end{array}$ \\
\hline CANMX & $\begin{array}{l}\text { Quantidade de água máxima interceptada pela vegetação } \\
(\mathrm{mm})\end{array}$ \\
\hline ESCO & $\begin{array}{l}\text { Coeficiente de compensação de evaporação de água no } \\
\text { solo (adimensional) }\end{array}$ \\
\hline SOL_ALB & Albedo do solo (adimensional) \\
\hline $\mathrm{CH} \mathrm{K}_{2}$ & Condutividade hidráulica efetiva do canal $\left(\mathrm{mm} \mathrm{h}^{-1}\right)$ \\
\hline $\mathrm{BIOM}$ & Eficiência da mistura biológica do solo (adimensional) \\
\hline
\end{tabular}

A calibração de um modelo hidrológico é uma etapa fundamental e consiste na modificação dos parâmetros dentro de um intervalo aceitável, para obtenção de um modelo que replique condições previamente conhecidas do processo natural modelado (Viessman \& Lewis, 2003). O SWAT inclui uma função multiobjetiva para o procedimento de calibração automática desenvolvido por Griensven \& Bauwens (2003). O procedimento de calibração é baseado no algoritmo Shuffled Complex Evolution (SCE-UA) desenvolvido na Universidade do Arizona e é descrito por Duan et al. (1992).

No início da simulação ocorrem incertezas devido ao desconhecimento das condições iniciais, principalmente da umidade do solo, justificando a utilização de um período de aquecimento do modelo para que, ao se iniciar a simulação, as variáveis de estado estejam livres da influência das condições iniciais (Mello et al., 2008). No presente trabalho foi utilizado o período de 1 de janeiro a 31 de agosto de 2006, para o aquecimento do modelo SWAT cujo processo de calibração se deu no período de 1 de setembro de 2006 a 31 de agosto de 2008 .

Os modelos hidrológicos são calibrados com dados de um período de tempo e verificados com dados de outro período, processo este conhecido como validação.Para isto e com os parâmetros previamente calibrados, o modelo foi aplicado ao período de 01 de setembro de 2008 a 31 de agosto de 2009. De acordo com Arabi et al. (2006) esta etapa de validação permite confirmar a acurácia do modelo visando à simulação de processos estacionários em bacias hidrográficas com monitoramento hidrológico.

Para avaliar o desempenho do modelo tanto na fase de calibração quanto na de validação, foram utilizadas as seguintes estatísticas de precisão: coeficiente de Nash-Sutcliffe $\left(\mathrm{C}_{\mathrm{NS}}\right)$ (Nash \& Sutcliffe, 1970) e $\mathrm{P}_{\text {BIAS }}$ (Kumar, 2008).

$\mathrm{O} \mathrm{C}_{\mathrm{NS}}$ (Eq. 2) traduz a eficiência da aplicação do modelo para previsões mais acertadas das vazões de cheia. Santhi et al. (2001) apresentam a seguinte classificação para este coeficiente: $\mathrm{C}_{\mathrm{NS}}>0,65$ o modelo é considerado muito bom; $0,54<\mathrm{C}_{\mathrm{NS}}<$ 0,65 o modelo é considerado bom e entre 0,5 e 0,54 , satisfatório.

$\mathrm{O} \mathrm{P}_{\mathrm{BIAS}}$ (Eq. 3) diz respeitoao percentual de vies das vazões simuladas em relação às observadas. Quanto mais próximo de zero for o valor deste coeficiente melhor o modelo representará a realidade, ou seja, menor a tendência nas estimativas e, além disso, serve também como indicativo se o modelo é pobre em representatividade (Moriasi et al., 2007). Liew et al. (2007) apresentaram a seguinte classificação: $\left|\mathrm{P}_{\mathrm{BIAS}}\right|<10 \%$, muito bom; $10 \%<\left|\mathrm{P}_{\text {BIAS }}\right|<15 \%$, bom; $15 \%<\left|\mathrm{P}_{\text {BIAS }}\right|<25 \%$, satisfatório e $\left|\mathrm{P}_{\text {BIAS }}\right|>25 \%$, o modelo é inadequado.

$$
\begin{gathered}
\mathrm{C}_{\mathrm{NS}}=1 \frac{\sum_{\mathrm{i}=1}^{\mathrm{n}}\left(\mathrm{Q}_{\mathrm{obs}_{\mathrm{i}}}-\mathrm{Q}_{\text {sim }_{\mathrm{i}}}\right)^{2}}{\sum_{\mathrm{i}=1}^{\mathrm{n}}\left(\mathrm{Q}_{\mathrm{obs}_{\mathrm{i}}}-\overline{\mathrm{Q}}_{\mathrm{obs}}\right)^{2}} \\
\mathrm{P}_{\text {BIAS }}=\left(\frac{\overline{\mathrm{Q}}_{\text {sim }}-\overline{\mathrm{Q}}_{\mathrm{obs}}}{\overline{\mathrm{Q}}_{\mathrm{obs}}}\right) \times 100
\end{gathered}
$$


$\mathrm{Q}_{\mathrm{OBSi}}$ - vazão observada

$\mathrm{Q}_{\mathrm{SIMi}}$ - vazão simulada

$\overline{\mathrm{Q}}_{\mathrm{OBS}}$ - vazão média observada

n - número de eventos

Complementarmente aos estudos estatísticos realizaramse análises sobre informações frequentemente extraídas do hidrograma, em especial algumas associadas à curva de permanência de vazões.

\section{RESUlTADOS E DISCUSSÃO}

Na Tabela 2 estão listados os parâmetros considerados mais sensíveis, os processos hidrológicos aos quais estão relacionados e suas descrições dentro do modelo SWAT. Além de caracterizar os parâmetros mais sensíveis e passíveis de calibração, a análise de sensibilidade também é imprescindível para verificar se o modelo está capturando, de forma correta, o comportamento dos processos hidrológicos predominantes na bacia. Outro aspecto a ser mencionado é que a análise de sensibilidade é extremamente dependente da estatística de precisão utilizada para quantificar a sensibilidade, ou seja, o $\mathrm{C}_{\mathrm{NS}}$ certamente sofrerá mais influência de parâmetros vinculados ao escoamento superficial direto. Os parâmetros mais sensíveis, listados na Tabela 2, estão de acordo com a predominância dos processos hidrológicos na BHRJ, ocorrendo predominância de escoamento de base, seguido do escoamento superficial direto.

Alguns dos parâmetros identificados como mais sensíveis não são facilmente mensuráveis ou estimáveis para a BHRJ devido ao fato de que valores realísticos não estão disponíveis; apesar disto, parâmetros mensurados direta ou indiretamente por meio de amostragens representativas realizadas "in situ", tais como SOL_AWC, SOL_K e SOL_Z, foram incluídos na calibração visto que a análise de sensibilidade mostrou que eles influenciaram consideravelmente a resposta do modelo. É oportuno ressaltar que esta é uma limitação importante do modelo SWAT haja vista que não permite que dados distribuídos no espaço da bacia, associados aos atributos físicos do solo (armazenamento de água e condutividade hidráulica) sejam alimentados no modelo, na forma de mapas; contudo, essas variáveis hidrológicas são altamente sensíveis no tocante à geração do escoamento superficial direto e, consequentemente, em todo o balanço hídrico executado pelo modelo.

Os parâmetros calibrados e suas respectivas faixas de variação estão apresentados na Tabela 3.

Tabela 3. Parâmetros utilizados na calibração do modelo SWAT com os valores calibrados

\begin{tabular}{cccc}
\hline \multirow{2}{*}{ Parâmetro } & \multicolumn{2}{c}{ Intervalo do valor do parâmetro } & Valor calibrado \\
\cline { 2 - 3 } CN2 & Inferior & Superior & \\
ALPHA_BF & $-50 \%$ & $50 \%$ & $-38,18 \%$ \\
RCHRG_DP & 0 & 1 & 0,01 \\
ESCD & 0 & 1 & 0,484 \\
SOL_Z & 0 & 1 & 0,043 \\
SOL_K & $-50 \%$ & $50 \%$ & $-49,75 \%$ \\
SOL_AWC & $-50 \%$ & $50 \%$ & $50,00 \%$ \\
\hline
\end{tabular}

Na Tabela 3 pode-se constatar que alguns parâmetros são não apenas calibrados na forma percentual (CN2, Sol_Z, Sol_K e Sol_AWC) e alterados de forma distribuída, embora sempre na mesma proporção. Outro comentário influente está associado à calibração do parâmetro SOL_K, cuja variação percentual atingiu o limite superior permitido pelo modelo. A justificativa está relacionada ao fato de que a condutividade hidráulica saturada possui alta variabilidade espacial e como no modelo não há distribuição espacial efetiva e, sim, uma média do seu valor para o correspondente tipo de solo e ainda que a variação permitida no modelo se ocorre de forma linear para

Tabela 2. Ranking de sensibilidade dos parâmetros utilizados na calibração do modelo SWAT, com os processos relacionados e sua descrição no modelo

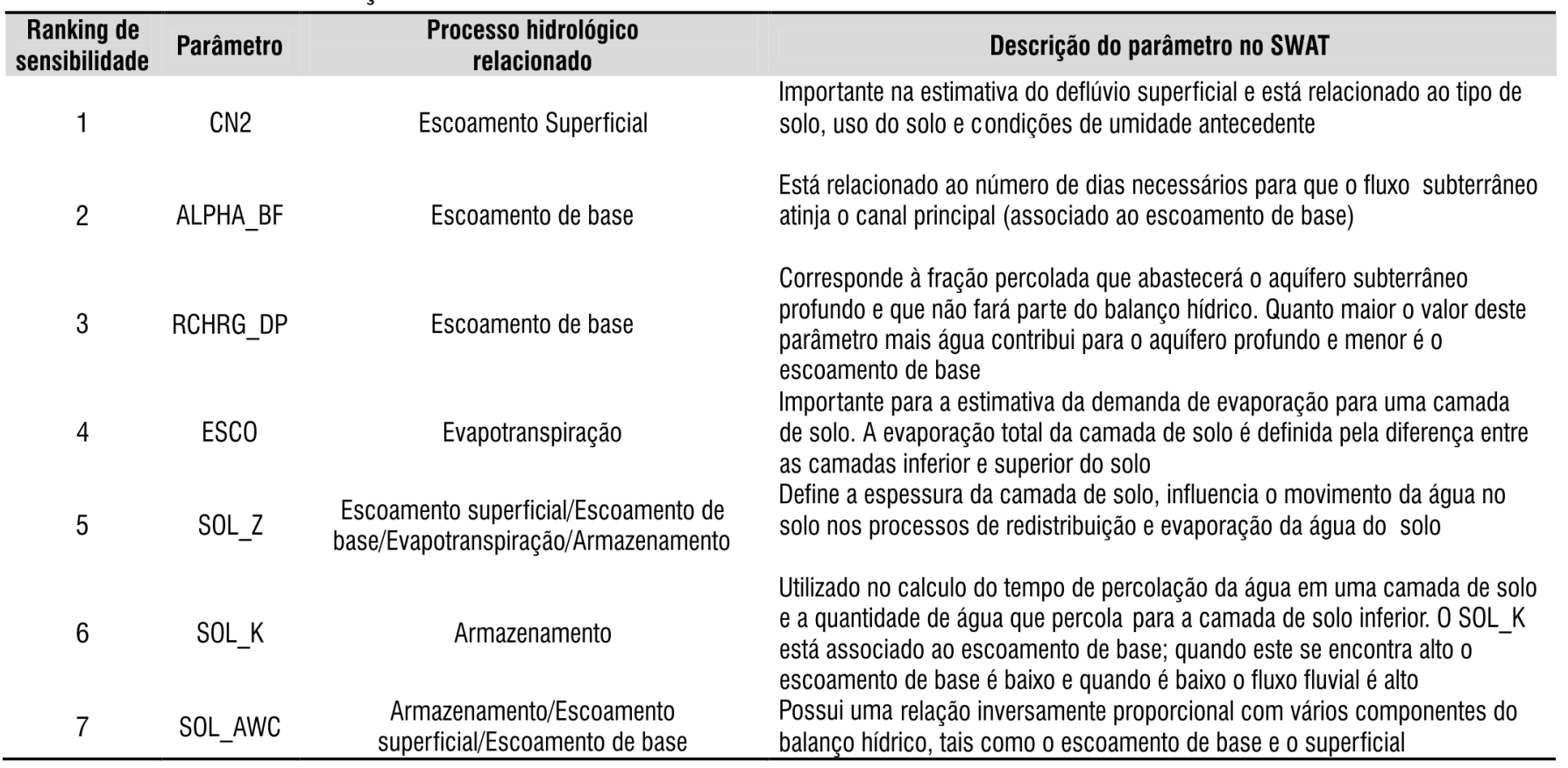


todas as HRU's, o modelo obteve, na busca da otimização, o valor máximo permitido no processo de calibração.

A análise visual do hidrograma simulado em relação ao observado consiste em uma importante ferramenta de avaliação do ajuste e essencial para uma correta avaliação do modelo. Nas Figuras 2A e 2B podem ser analisados os hidrogramas observado e simulado pelo modelo SWAT, nas fases de calibração e validação, respectivamente.

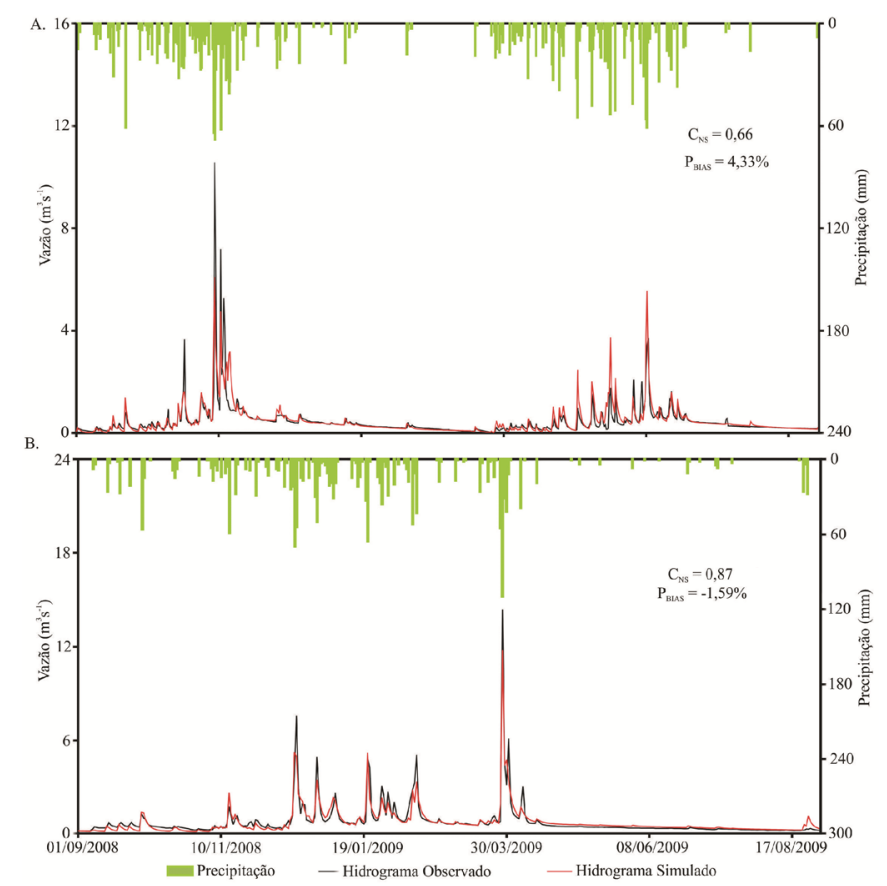

Figura 2. Hidrogramas observado e simulado para a BHRJ no período de calibração (A) e validação (B) e hietograma de precipitação diária na Bacia Hidrográfica do Ribeirão Jaguara (BHRJ)

Pode-se observar, nos períodos de recessão, que o ajuste do SWAT foi adequado, tanto na calibração quanto na validação, caracterizando o bom desempenho do modelo na simulação de vazões mínimas. Uma característica percebida no período de validação foi que a aderência do hidrograma simulado ao observado foi melhor quando comparada com a do período de calibração, refletindo em um $\mathrm{C}_{\mathrm{NS}}$ superior para a fase de validação.

Ao analisar o hidrograma observado na fase de calibração (Figura 2A) é possível observar grande concentração das vazões de pico no mês de janeiro de 2007 , fruto de intensa concentração da precipitação neste mês (583 $\mathrm{mm}$ para uma média de 230 $\mathrm{mm}$ ) com mais de 10 dias seguidos com precipitação diária superior a $35 \mathrm{~mm}$, situação que resulta em maior dificuldade para ajuste do modelo devido a uma resposta mais rápida da bacia aos eventos consecutivos de precipitação e, em contrapartida, picos seguidos de vazão. Durante o período utilizado para a validação do modelo SWAT (ano hidrológico 2008-2009), verifica-se, pelo hietograma da Figura 2B, que o período chuvoso (dezembro-março) não apresentou a mesma concentração de chuvas registrada em Janeiro de 2007 e, como consequência, menor concentração de picos seguidos de vazão, situação que facilita a aplicabilidade do modelo elevando o $\mathrm{C}_{\mathrm{NS}}$, visto que esta estatística é bastante dependente das estimativas de vazões de pico.
Os valores do coeficiente, Nash-Sutcliffe $\left(\mathrm{C}_{\mathrm{NS}}\right)$ o qual retrata a precisão do modelo sobretudo na estimativa de vazões de pico, foram 0,66 e 0,87 nas fases de calibração e validação, respectivamente. Tais resultados permitem enquadrar o modelo na categoria "muito bom" em ambas as fases, conforme classificação proposta por Santhi et al. (2001). O valor de $C_{N S}$ relativo à fase de validação foi notadamente maior que o valor obtido para calibração, aspecto este indicativo do bom ajuste do modelo à BHRJ.

São vastos os trabalhos na literatura que utilizaram o $\mathrm{C}_{\mathrm{NS}}$ para testar a eficiência do SWAT para simulação do comportamento de vazões em bacias hidrográficas. Pinto (2011) aplicou o modelo SWAT a uma bacia hidrográfica de cabeceira da Serra da Mantiqueira, com aproximadamente $7 \mathrm{~km}^{2}$, obtendo $\mathrm{C}_{\mathrm{NS}}$ para a calibração de 0,81 e para validação de 0,79 , sendo esses valores considerados muito bons. Sexton et al. (2010) obtiveram resultados de $\mathrm{C}_{\mathrm{NS}}$ para a calibração de duas bacias hidrográficas, localizadas nos Estados Unidos, entre 0,46 e 0,58 e, durante a validação, entre 0,68 e 0,78 , concluindo que os resultados encontrados foram aceitáveis e qualificaram o modelo SWAT para aplicação às bacias hidrográficas estudadas. Du et al. (2005) obtiveram, trabalhando em uma bacia do estado de Iowa (EUA) com dois postos fluviométricos diferentes, $\mathrm{C}_{\mathrm{NS}}$ para o período de calibração de 0,39 e 0,47 e, na validação, 0,32 e 0,35 .

Com base em diversos trabalhos sobre aplicação do modelo SWAT, em várias partes do mundo, pôde-se observar que os valores de $\mathrm{C}_{\mathrm{NS}}$ obtidos para a $\mathrm{BHRJ}$ foram bons o suficiente,de modo a qualificar o modelo apto à simulação hidrológica na referida bacia.

$\mathrm{O} \mathrm{P}_{\text {BIAS }}$ corresponde ao percentual de viés das vazões simuladas com relação às vazões observadas. Esta estatística de precisão apresentou pequenos desvios demonstrando ter ocorrido uma superestimativa da vazão na ordem 4,33\% no período de calibração e uma subestimativa de $-1,59 \%$ durante a validação. De acordo com Liew et al. (2007) este resultado indica boa adequação do modelo SWAT a esta bacia, ou seja, além de estimativas com boa precisão, dadas pelo coeficiente $\mathrm{C}_{\mathrm{NS}}$, elas não apresentaram tendência significativa nas estimativas significando que os parâmetros foram devidamente ajustados à BHRJ e não produziram estimativas enviesadas.

Os modelos hidrológicos podem ser úteis para uma gestão melhor de recursos hídricos, principalmente no contexto de bacias hidrográficas de pequeno porte. A verificação de vazões máximas, mínimas e de referência para outorga, propiciadas pela série simulada, possibilita a análise da aplicação do modelo como ferramenta de gestão e planejamento de recursos hídricos (Viola et al., 2009). Quando se trata da estimativa de vazões de referência para outorga há uma série de limitações para pequenas bacias, uma vez que é praticamente inexistente a disponibilidade de dados hidrológicos de vazão nesta escala no Brasil.A curva de permanência é uma das ferramentas para se determinar a disponibilidade hídrica em uma bacia hidrográfica, porém a mesma não se refere à probabilidade das vazões em qualquer ano, mas sim a uma "garantia" de ocorrência das vazões num horizonte de planejamento (Cruz \& Tucci, 2008). Na Figura 3 estão ilustradas as curvas de permanência observada e simulada pelo modelo, nas etapas de calibração (esquerda) e validação (direita). 


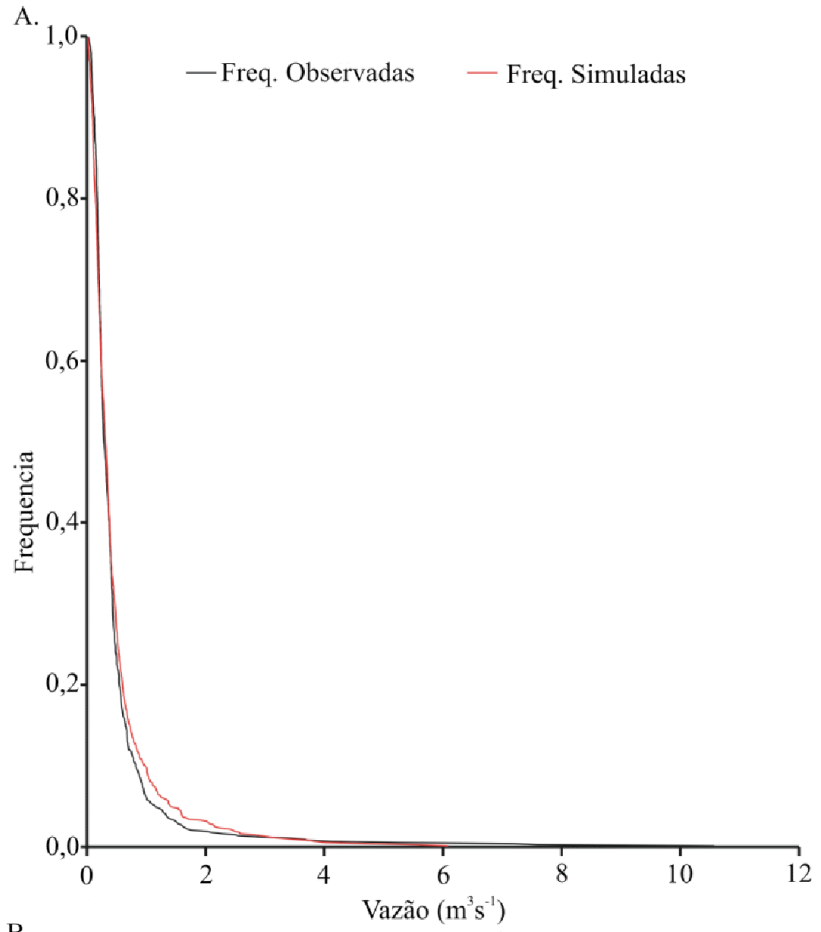

B.

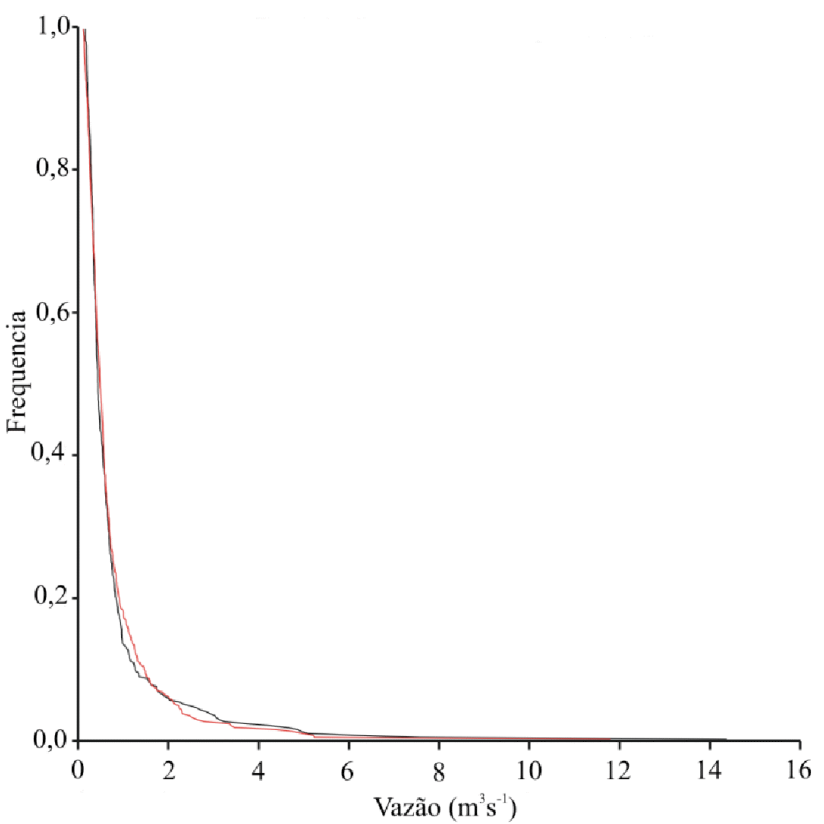

Figura 3. Curvas de permanência observada e simulada no período de calibração (A) e validação (B) do modelo SWAT para a Bacia Hidrográfica do Ribeirão Jaguara (BHRJ)

A análise visual das curvas de permanência permite a constatação de que tanto no período de calibração quanto no de validação, as vazões simuladas abaixo da permanência de $60 \%$ foram superestimadas, sobretudo nas permanências mais baixas. Com relação às vazões nas permanências superiores a 70\%, ou seja, as menores vazões, o modelo subestimou as vazões em até $33,14 \%$ na calibração e $20,49 \%$ na validação, erros que foram obtidos para a vazão com $95 \%$ de excedência $\left(Q_{95 \%}\right)$. Um valor de referência frequentemente extraído desta curva, é o de $90 \%$ de permanência $\left(\mathrm{Q}_{90 \%}\right)$ que representa a vazão superada ou igualada em $90 \%$ do tempo. Na curva observada da etapa de calibração o valor de $Q_{90 \%}$ foi de $0,122 \mathrm{~m}^{3} \mathrm{~s}^{-1}$ e na simulada foi de $0,091 \mathrm{~m}^{3} \mathrm{~s}^{-1}$ enquanto na validação o valor observado foi $0,219 \mathrm{~m}^{3} \mathrm{~s}^{-1}$ e o simulado $0,208 \mathrm{~m}^{3} \mathrm{~s}^{-1}$. Os resultados obtidos neste trabalho indicaram que o modelo subestimou a vazão de recessão em aproximadamente 24,90 e 4,80\%, na calibração e validação, respectivamente. Com base nesses erros, que em algumas permanências chegaram a 33,14\% mas que, de modo geral, foram de pequena magnitude para os demais valores, possibilitou constatar que o modelo SWAT simulou, de forma adequada, a curva de permanência de vazões para a BHRJ demonstrando sua qualidade como ferramenta auxiliar na gestão dos recursos hídricos.

\section{Conclusões}

1. O modelo SWAT foi calibrado e validado de forma adequada tendo-se obtido valores de $\mathrm{C}_{\mathrm{NS}}$ de 0,660 e 0,872 para os períodos de calibração e validação, respectivamente.

2. O modelo SWAT, como adequado e apto à simulação hidrológica na bacia hidrográfica estudada.

3. As vazões máximas, mínimas e aquelas associadas a diferentes permanências, em especial, a $\mathrm{Q}_{90 \%}$, atestaram a aplicabilidade do modelo como ferramenta de gestão e planejamento dos recursos hídricos em bacias hidrográficas representativas dos latossolos na região do Alto Rio Grande, MG.

\section{LITERATURA CITADA}

Arabi, M.; Govindaraju, R. S.; Hantush, M. M. A probabilistic approach for analysis of uncertainty in the evaluation of watershed management practices. Journal of Hydrology, v.333, p.459-471, 2006.

Araújo, A. R. Solos da Bacia do Alto Rio Grande: Base para estudos hidrológicos e aptidão agrícola. Lavras: UFLA, 2006. 332p. Tese Doutorado

Arnold, J.G.; P. M. Allen. Estimating hydrologic budgets for three Illinois watersheds. Journal of Hydrology, v.176, p.57-77, 1996.

Baltokoski, V.; Tavares, M. H. F.; Machado, E.; Oliveira, M. P. de, Calibração de modelo para a simulação de vazão e de fósforo total nas sub-bacias dos Rios Conrado e Pinheiro - Pato Branco (PR). Revista Brasileira de Ciência do Solo, v.34, p.253-261, 2010.

Beskow, S.; Mello, C. R. de; Norton L. D. Development, sensitivity and uncertainty analysis of LASH model. Scientia Agricola, v.68, p.265-274, 2011a.

Beskow, S.; Mello, C. R.; Norton, L. D.; Silva, A. M. Performance of a distributed semi-conceptual hydrological model under tropical watershed conditions. Catena, v.86, p.160-171, $2011 \mathrm{~b}$.

Collishonn, W.; Allasia, D. G.; Silva, B. C.; Tucci, C. M. The MGB-IPH model for large-scale rainfall-runnof modeling. Hydrological Science Journal, v.52, p.878-895. 2007.

Cruz, J. C.; Tucci, C. E. M. Estimativa da disponibilidade hídrica através da curva de permanência. Revista Brasileira de Recursos Hídricos, v.13, p.111-124, 2008. 
Du, B.; Arnold, J. G.; Saleh, A.; Jaynes D. B. Development and application of SWAT to landscapes with tiles and potholes. Transaction of the ASAE, v.48, p.1121-1133, 2005.

Duan, Q.; Sorooshian, S.; Gupta, V. Effective and efficient global optimization for conceptual rainfall-runoff models. Water Resources Research, v.28, p.1015-1031, 1992.

Durães, M.F.; Mello, C.R.; Naghettini, M. Applicability of the SWAT model for hydrologic simulation in Paraopeba River Basin, MG. Cerne, v.17, p.481-488, 2011.

Gassman, P. W.; Reyes, M. R.; Green C. H.; Arnold, J.G. The soil and water assessment tool: historical development, applications, and future research directions. Transaction of the ASABE, v.50, p.1211-1250, 2007.

Gomes, N. M.; Faria, M. A. de; Silva, A. M.; Mello, C. R. de; Viola, M. R. Variabilidade espacial de atributos físicos do solo associados ao uso e ocupação da paisagem. Revista Brasileira de Engenheira Agrícola Ambiental, v.11, p.427$435,2007$.

Griensven, A. Sensitivity, auto-calibration, uncertainty and model evaluation in SWAT 2005.http://groups.google.com/ group/swatuser/files. 3 Jul. 2011.

Griensven, A.; Bauwens, W. Concepts for river water quality processes for an integrated river basin modeling. Water Science and Technology, v.48, p.1-8, 2003.

Kumar, S. Studying the effect of spatial scaling on hydrologic model calibration using soil and water assessment tool (SWAT). West Lafayette: Purdue University, 2008. 124p. Dissertação Mestrado

Liew, M. W.; Veith, T. L.; Bosch, D. D.; Arnold, J. G. Suitability of SWAT for the Conservation effects assessment project: A comparison on USDA-ARS watersheds. Journal of Hydrological Research, v.12, p.173-189, 2007.

Marchioro, E. Modelagem hidrossedimentológica na bacia do córrego Santa Maria: Subsídios à aplicação de práticas de conservação de água e solo no noroeste fluminense. Rio de Janeiro: UFRJ, 2008. 189p. Tese Doutorado

Mello, C. R.; Norton, L. D.; Curi, N.; Yanagi, S. N. M. Sea surface temperature (SST) and rainfall erosivity in the Upper Grande River Basin, Southeast Brazil. Ciência e Agrotecnologia, v.36, p.53-59, 2012.

Mello, C. R. de; Viola, M. R.; Norton, L. D.; Silva, A. M.; Acerbi Júnior, F. W. Development and application of a simple hydrologic model simulation for a Brazilian head water basin. Catena, v.75, p.235-247, 2008.

Moriasi, D. N.; Arnold, J. G.; Liew, M. W. van; Binger, R. L.; Harmel, R. D.; Veith, T. L. Model evaluation guidelines for systematic quantification of accuracy in watershed simulations. Transactions of the ASABE, v.50, p.885-900, 2007.

Muleta, M. K.; Nicklow, J. W. Sensitivity and uncertainty analysis coupled with automatic calibration for a distributed watershed model. Journal of Hydrology, v.306, p.127-145, 2005.
Nash, J.E.; Sutcliffe, J.V. River flow forecasting through conceptual models Part I - A discussion of principles. Journal of Hydrology, v.10, p.282-290, 1970.

Neitsch, S. L.; Arnold, J. R.; Kiniry, J. R.; Williams, J. J. Soil and water assessment tool: theoretical documentation. Versão 2005. Grassland: Temple, 2005. 525p.

Pinto, D. B. F. Aplicação do modelo SWAT (Soil and Water Assessment Tool) na simulação hidrossedimentológica em bacia hidrográfica da Serra da Mantiqueira, MG. Lavras: UFLA, 2011. 225p. Tese Doutorado

Santhi, C.; Arnold, J. G.; Williams, J. R.; Dugas, W. A.; Sirinivasan, R.; Hauck, L. M. Validation of the SWAT model on a large river basin with point and nonpoint sources. Journal of the American Water Resources Association, v.37, p.1169-1188, 2001.

Saraiva, I.; Fernandes, W.; Naghettini, M. Simulação hidrológica mensal em bacias sem monitoramento fluviométrico. Revista Brasileira de Recursos Hídricos, v.16, p.115-125, 2011.

Sexton, A. M.; Sadeghi, A. M.; Zhang X.; Srinivasan, R.; Shirmohammadi, A. Using NEXRAD and rain-gauge precipitation data for hydrologic calibration of SWAT in northeastern watershed. Transactions of the ASABE, v.53, p.1501-1510, 2010.

Silva, V. A.; Moreau, M. S.; Moreau, A. M. S. dos; Rego, N. A. C. Uso da terra e perda de solo na bacia hidrográfica do rio colônia, Bahia. Revista Brasileira de Engenharia Agrícola e Ambiental, v.15, p.310-315, 2011.

Spruill, C. A.; Workman, S. R.; Taraba, J. L. Simulation of daily and monthly stream discharge from small watersheds using the SWAT model. Transactions of the ASAE, v.43, p.1431-1439, 2000.

Veith, T. L.; Liew, M. W. van; Bosch, D. D.; Arnold, J. G. Parameter sensitivity and uncertainty in SWAT: A comparison across five USDA-ARS watersheds. Transactions of the ASABE, v.53, p.1477-1486, 2010.

Viessman, W.; Lewis, G. L. Introduction to hydrology. 5.ed. New Jersey: Upper Saddle River. 2003. 612p.

Vieux, B. E. Distributed hydrologic modeling using GIS. 2.ed. Dordrecht: Kluwer Academic Publishers, 2004. 289p.

Viola, M. R.; Mello, C. R. de; Acerbi Júnior, F. W.; Silva, A. M. Modelagem hidrológica na Bacia Hidrográfica do Rio Aiuruoca, MG. Revista Brasileira Engenheira Agrícola e Ambiental, v.13, p.581-591, 2009.

Wagener, T.; Wheater, H.; Gupta, H. V. Rainfall-runoff modeling in gauged and ungauged catchments. 1.ed. Londres: Imperial College, 2004. 306p.

Yuan, Y.; Binger, R. L.; Locke, M. A.; Theurer, F. D.; Stafford, J. Assessment of subsurface drainage management practices to reduces nitrogen loadings using AnnAGNPS. Applied Engineering in Agriculture, v.27, p.335-344, 2011. 